\title{
Palmar and plantar fibromatosis: a review
}

\author{
Brian D. Stewart ${ }^{1}$, Alessandra F. Nascimento ${ }^{2}$ \\ 'Department of Pathology, Immunology and Laboratory Medicine, University of Florida College of Medicine, Gainesville, FL; \\ ${ }^{2}$ CWRU School of Medicine, University Hospitals, Bone and Soft Tissue Pathology, Cleveland, OH, USA
}

Palmar fibromatosis (Dupuytren disease/contracture) is the most common type of fibromatosis, defined as a benign proliferation of fibroblasts and myofibroblasts. The disease process is most common in white, middle-aged and older men occurring at the distal palmar crease leading to nodules and contracture, which in many cases recur after surgical treatment. In a similar process, plantar fibromatosis (Ledderhose disease) is a proliferation of fibroblasts and myofibroblasts on the plantar aponeurosis of mostly middle-aged patients that may lead to painful nodules but usually does not lead to contracture. Both processes are histologically similar, composed of a bland cellular proliferation of spindle cells with a bluish appearance and with a variable amount of background collagen, depending on the age of the lesion. The etiology of both lesions is still uncertain, while treatment ranges from observation to surgery, with some pharmacologic agents being investigated with mixed success. In this paper we provide an overview of both processes with regards to clinical and radiologic findings, pathophysiology, diagnosis, treatment, and prognosis.

Key Words: Fibromatosis; Plantar; Palmar; Dupuytren; Ledderhose

Received: June 5, 2021 Accepted: June 14, 2021

Corresponding Author: Brian D. Stewart, MD, Department of Pathology, Immunology and Laboratory Medicine, University of Florida College of Medicine, P.0. Box 100275,

1600 SW Archer Road, Gainesville, FL 32610-0275, USA

Tel: +1-352-627-9240, Fax: +1-352-627-9242, E-mail: bstewart@ufl.edu

This article has been published jointly, with consent, in both Journal of Pathology and Translational Medicine and PathologyOutlines.com.

Palmar and plantar fibromatoses are both benign, superficial, proliferative processes of the palmar and plantar aponeuroses, respectively, caused by the proliferation of fibroblasts and myofibroblasts. Other terms used for this in the clinical setting are Dupuytren disease or contracture for palmar fibromatosis and Ledderhose disease or morbus Ledderhose for plantar fibromatosis. This review will offer insights into the epidemiology, clinical features, pathophysiology, imaging characteristics, treatment, and prognosis of these common entities.

\section{ETIOLOGY AND PATHOGENESIS}

Palmar fibromatosis is the most common type of superficial fibromatosis affecting approximately $1 \%-2 \%$ of the population. The prevalence increases with the patient's age to where approximately $20 \%$ of the population at age 65 suffers from this disease. Males are more often affected, with a male to female ratio of 3-4:1. It can affect both the metacarpophalangeal and proximal interphalangeal joints [1]. Half of the cases affect both hands.
Patients of northern European ancestry are most commonly affected and the disease is rare in the black population.

Palmar fibromatosis is associated with diabetes, smoking, repetitive vibrational trauma and is thought to be caused by fibrogenic cytokines [2]. The process is characterized by a proliferation of fibroblasts followed by their differentiation into myofibroblasts and the production of extracellular matrix. Fibroblast growth factor, wingless/integrated (Wnt), and transforming growth factor $\beta$ have all been proposed as having a role in disease progression [3-7].

The prevalence of plantar fibromatosis is not currently well characterized and is now on the National Institutes of Health list of rare diseases [8]. The disease process usually affects the medial and central bands of the plantar aponeurosis of middleaged patients, although several reports of patients less than 16 years old to as young as 9 months have been published [9]. Approximately $25 \%$ of cases are bilateral with males being more affected than females [10]. A possible genetic predisposition has been suggested, as two genetic variants were found in a genome 
wide association study where one indel (chr5:118704153:D) and one single nucleotide polymorphism (rs62051384) were detected [11].

The etiology of plantar fibromatosis is not currently understood, but has been associated with long-term phenobarbital use for epilepsy, frozen shoulder, smoking, alcohol addiction, diabetes, and repeated trauma $[8,12]$. The disease process advances through several phases. There is first the proliferative phase in which increased fibroblastic activity and cellular proliferation is seen. This is followed by the active phase where nodule formation occurs. Finally, there is the residual phase consisting of collagen deposition, scar formation and tissue contracture.

\section{CLINICAL FEATURES AND RADIOLOGY}

The diagnosis of both plantar and palmar fibromatosis is usually made clinically, although occasionally histologic confirmation may be necessary. Palmar fibromatosis often presents with subcutaneous nodules on the distal volar aspect which puckers the overlying skin as it ages. The process leads to painful flexion contracture, most commonly of digits 4 and 5, due to cord-like expansion of the digital aponeurotic slips (Fig. 1A, B). Deeper structures such as the tendons or skeletal muscle are not involved. Plantar disease is concurrently seen in $10 \%$ of patients, while an additional 1\%-4\% have penile fibromatosis (Peyronie disease). Ultrasound of the hand shows nodules superficial to the flexor tendons in superficial fascia. The early lesions are hypoechoic with hypervascularity while more chronic lesions become more hyperechoic without vascularity [13]. The subcutaneous nodules on magnetic resonance imaging (MRI) are typically uniformly of low signal intensity on both T1 and T2 [14].

Plantar fibromatosis presents with single or multiple slow growing subcutaneous nodules located in the medial or central plantar aponeurosis measuring 0.5 to $3.0 \mathrm{~cm}$ in diameter. These are initially painless but are later associated with pain after standing or walking, typically on the medial aspect of the sole. Plantar disease is associated with concomitant palmar and penile fibromatoses, along with keloids. Unlike palmar disease, plantar fibromatosis is usually not associated with contractures [15]. Radiographs of the foot are usually normal. Ultrasonography is seen as superior to MRI, where hypoechogenic to mixed lesions are embedded on the plantar fascia and less reflective to the much brighter plantar fascia surrounding it with sharp juxtaposition [16]. The plantar fascia shows discrete, fusiform, multinodular thickening [17]. Alternating linear bands of hypoechogenicity and isoechogenicity relative to the plantar fascia, known as the Comb sign, is seen in $51 \%$ of cases [18]. MRI demonstrates nodules that appear as focal oval-shaped areas of disorganization embedded in the plantar fascia [16]. T1-weighted images show isometric to low signal intensity as compared to muscle, while T2weighted MRI shows low to intermediate signal $[17,19]$.

\section{PATHOLOGIC FINDINGS}

Palmar fibromatosis presents macroscopically as small nodules or nodular masses associated with aponeuroses and subcutaneous fat (Fig. 2) with a gray to yellow to white cut surface, the exact nature of the color depending on the amount of collagen content. Cytologic examination is usually limited to touch preparations made during the rare specimen sent for frozen section (Fig. 3A, B). These are usually hypocellular with clusters of bland spindle cells with oval to elongated nuclei. Nuclear atypia and mitotic activity are not present. The microscopic features depend on the age of the lesion. In the proliferative phase, there are uniform,
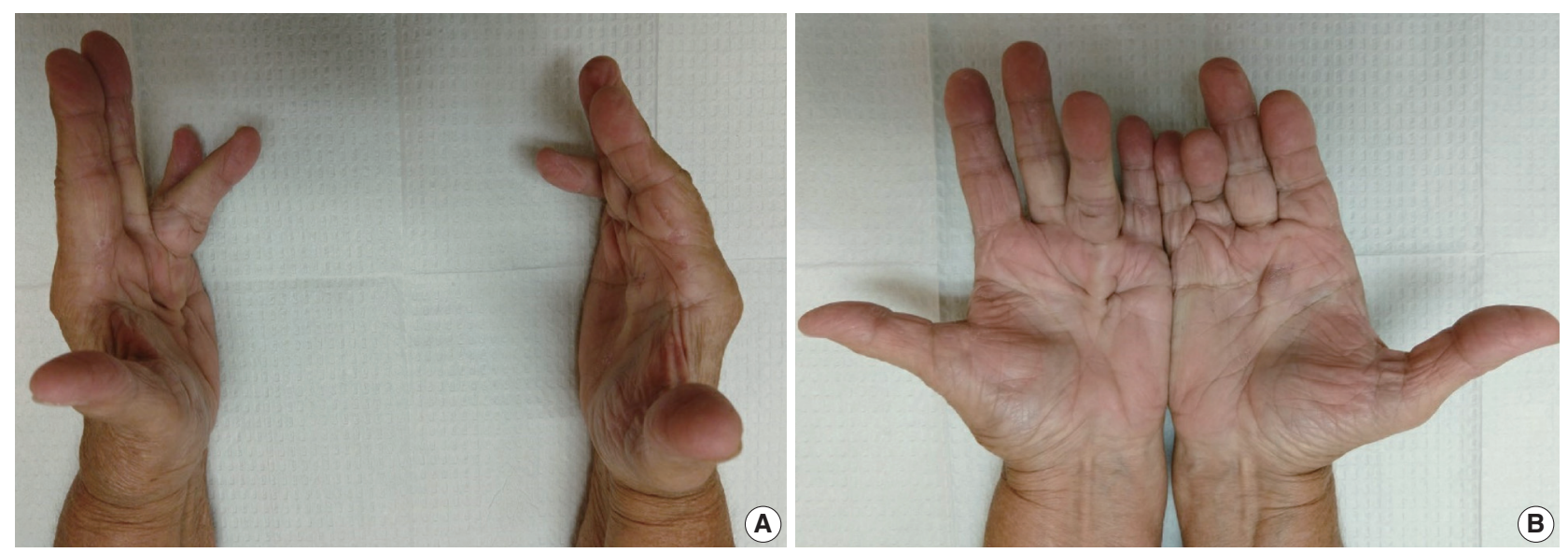

Fig. 1. (A, B) Bilateral nodules in the distal palmar crease and contractures involving the fourth digit. 
plump, spindle cells (myofibroblasts and fibroblasts) with bland nuclei and indistinct nucleoli, usually with a "bluer" appearance than the surrounding aponeurotic tissue (Figs. 4-6). The stroma contains a moderate amount of collagen and elongated vessels. In older, less cellular lesions, the collagenous content is denser. Occasionally, attachment to the overlying dermis or cartilaginous metaplasia can be seen. There is usually no infiltration into surrounding tissue beyond the subcutis.

The gross and microscopic characteristics of plantar fibromatosis are similar to its palmar counterpart (Figs. 7-9). Evans described the presence of a variable number of multinucleated giant cells found during the proliferative phase [20]. The formation of nodules is seen during the active phase while the less cellular, more collagenous residual phase often has a prominent chronic inflammatory component and hemosiderin deposition.

Immunohistochemical studies are usually not necessary for di-

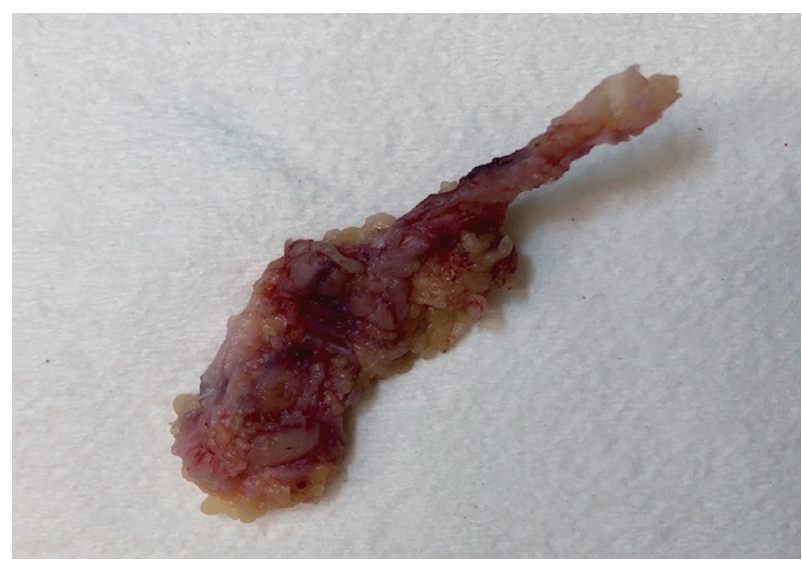

Fig. 2. Excised contraction band of palmar fibromatosis with surrounding soft tissue.

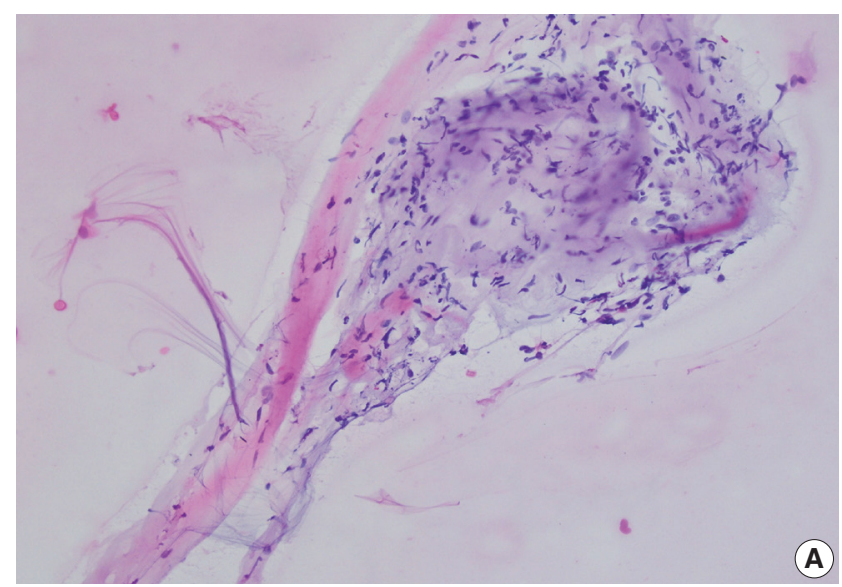

agnosis due to the characteristic histology of these lesions. Vimentin is uniformly reactive while muscle specific and smooth muscle actins are variable. Infrequently, desmin may show reactivity. Keratins, CD34, epithelial membrane antigen, and S-100 should all be negative. Beta-catenin is negative in plantar fibromatosis, but aberrant nuclear staining in palmar fibromatosis is common $[21,22]$.

\section{MOLECULAR AND CYTOGENETICS}

Palmar fibromatosis is usually considered a reactive, as opposed to neoplastic, lesion [23]. The lesions are near diploid, often with trisomy 7 or 8 , and show no gene amplifications or deletions [24]. Loss of the $Y$ chromosome may be seen [25]. Aberrations in the Wnt signaling pathway are common; however, no somatic mutations of beta-catenin genes, as seen in desmoid fibromatosis, are present $[6,22,26,27]$.

Like palmar fibromatosis, plantar fibromatosis is near diploid, often with trisomy 7 or 8 , and does not contain somatic mutations of beta-catenin genes [22]. Trisomy 14 has also been reported, along with a case containing a clonal reciprocal $\mathrm{t}(2 ; 7)$ (p13;p13) $[28,29]$.

\section{DIFFERENTIAL DIAGNOSIS}

The clinical, gross, and histologic features of palmar and plantar fibromatosis are quite characteristic and the diagnosis is usually straightforward. However, it is important to keep in mind some of the differential diagnoses. Spindle cell sarcomas, such as synovial sarcoma and malignant peripheral nerve sheath tumor (MPNST), and epithelioid sarcoma should not be missed as the

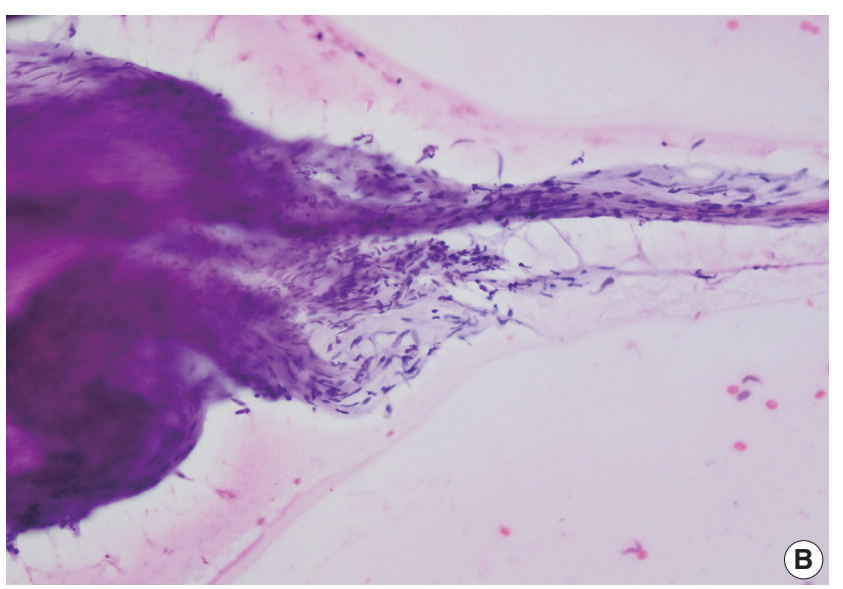

Fig. 3. (A, B) Touch prep of a specimen sent for frozen section showing clusters of loosely arranged spindle cells with bland fusiform nuclei without atypia or mitotic figures. 
treatment and prognosis is vastly different. Epithelioid sarcoma commonly presents in the hand but cells will show a distinctive epithelioid appearance with abundant eosinophilic cytoplasm.

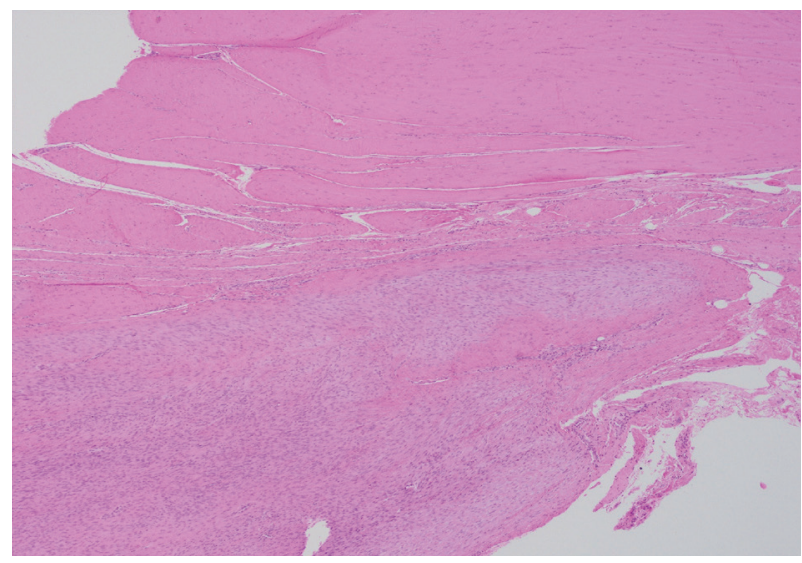

Fig. 4. Immature fibroblastic proliferation (lower 1/2) well-demarcated from the involved tendon (upper 1/2).

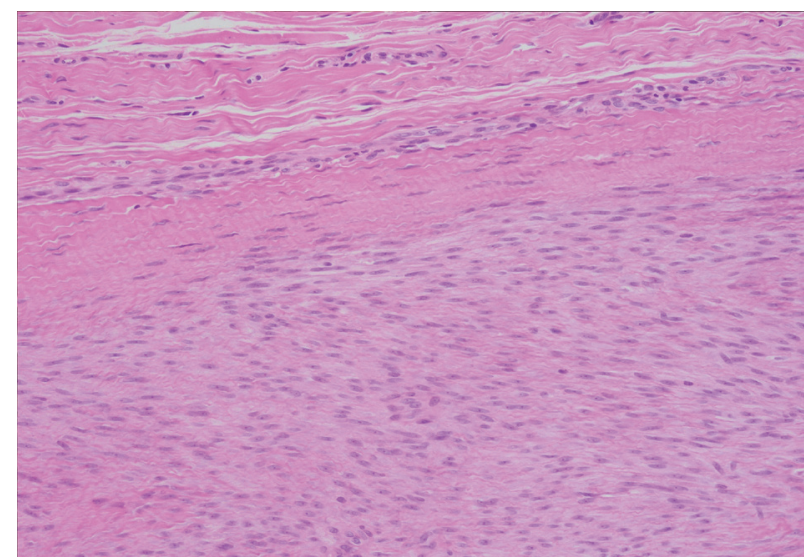

Fig. 5. Higher magnification showing the immature fibroblastic proliferation (lower $2 / 3$ of the field) involving normal fibrous tissue (upper 1/3).

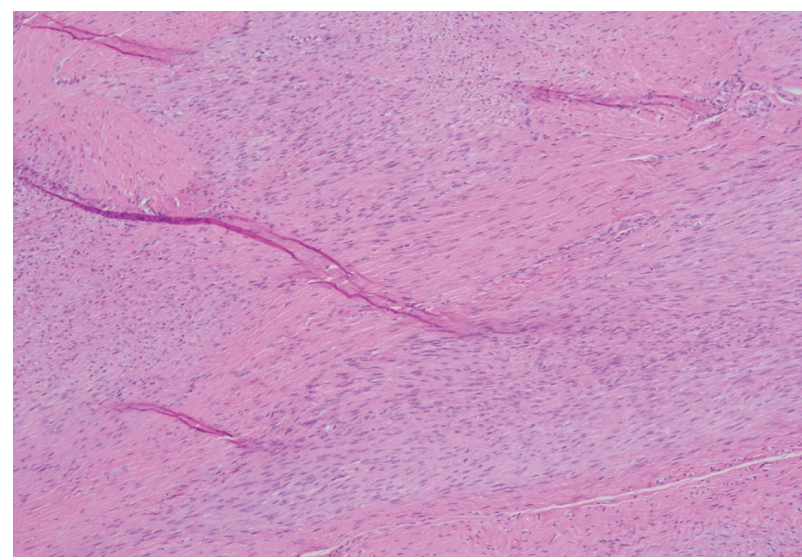

Fig. 6. Tendinous tissue with interspersed fascicles of a bland appearing immature spindle cell proliferation in both longitudinal and cross sections.
Central necrosis and/or hyalinization is often seen. Epithelioid sarcoma is characteristically reactive for cytokeratins and CD34 and shows a loss of nuclear staining for SMARCB1 (INI-1).

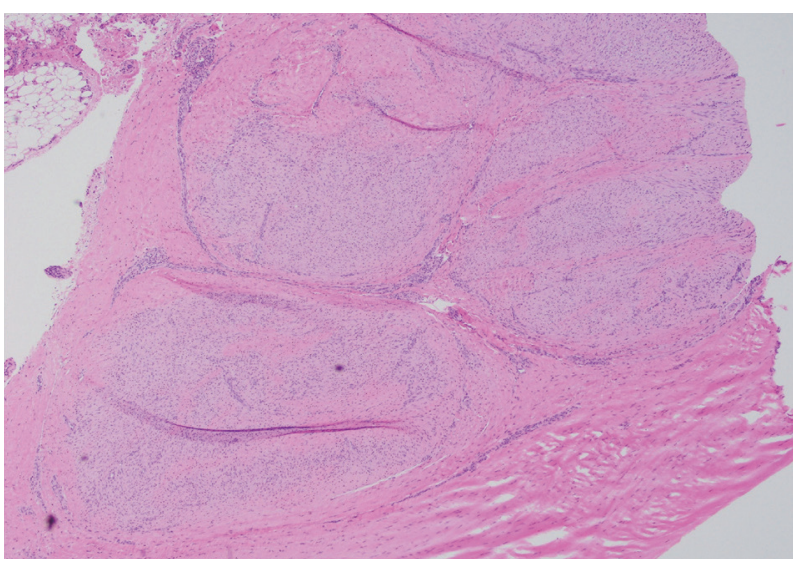

Fig. 7. Nodular proliferation of immature fibroblasts and myofibroblasts embedded within the plantar aponeurosis.

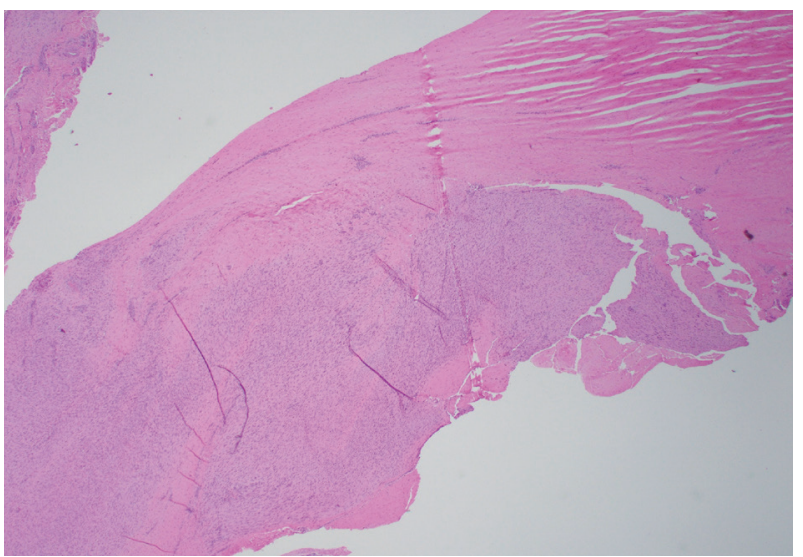

Fig. 8. Large nodule of immature fibroblasts and myofibroblasts (lower 1/2) with pushing and locally infiltrating borders into the plantar aponeurotic tissue (upper 1/2).

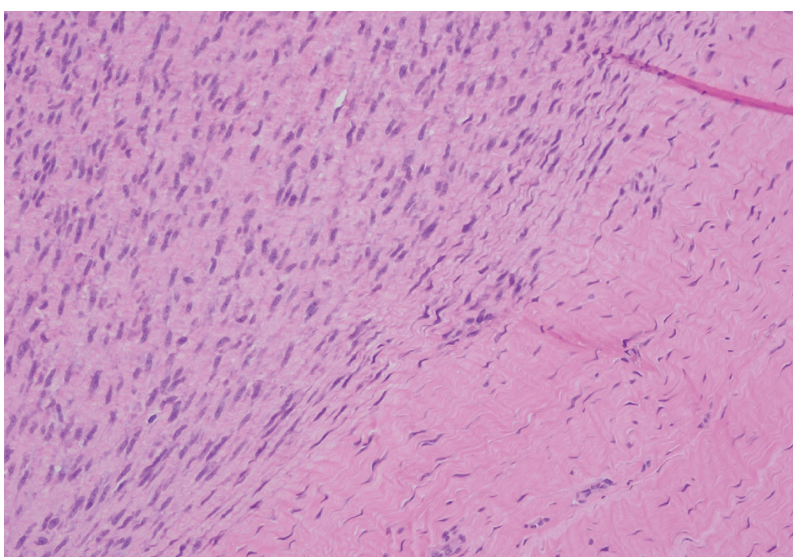

Fig. 9. Cellular proliferation of immature fibroblasts and myofibroblasts with plump to fusiform nuclei (left) well demarcated from the less cellular and more collagenous plantar fascia (right). 

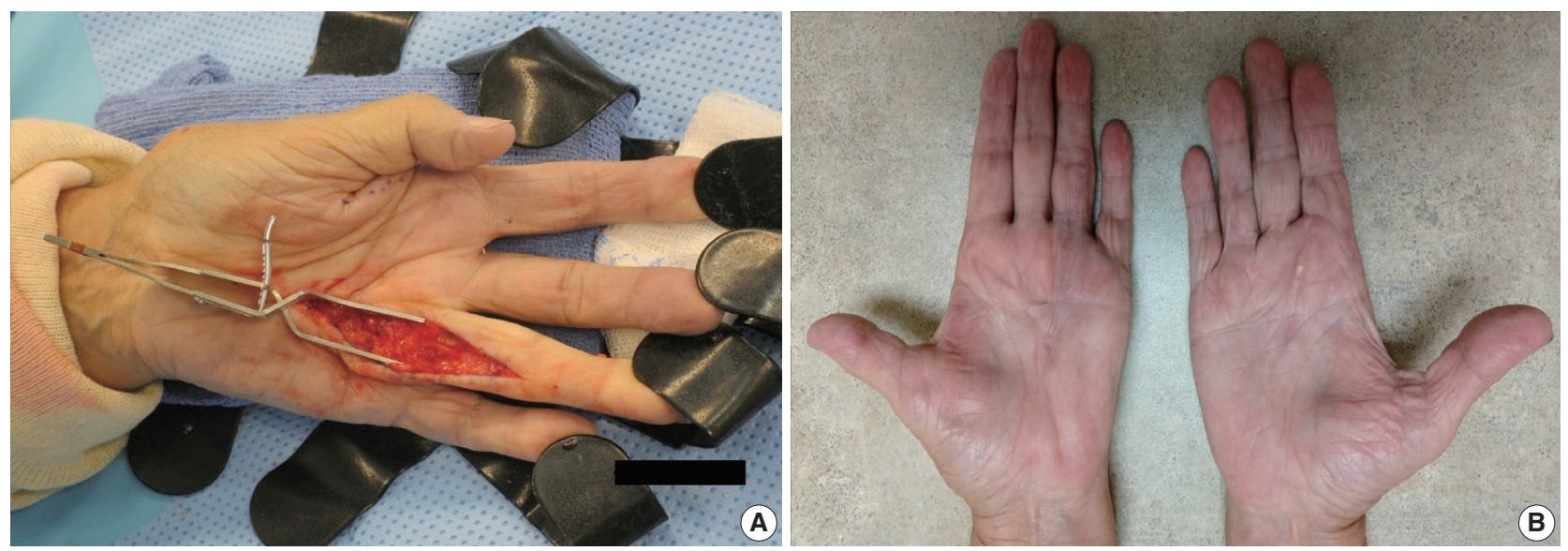

Fig. 10. (A, B) Patient from Fig. 1 after removal of the contraction band and resolution of the contracture of the fourth digits.

Clinically, plantar fibromatosis that has not formed nodules may be confused with a calcaneal stress fracture, tarsal tunnel syndrome, or plantar fasciitis [10]. With the development of nodules, malignancies such as melanoma, synovial sarcoma, and Kaposi sarcoma may be of concern. On a histologic level, calcifying aponeurotic fibroma can be excluded as plump or epithelioid fibroblasts palisading around cartilage and spotty calcification is seen in this entity and not in plantar fibromatosis. As in the hand, desmoid-type fibromatosis is rare in the feet and infiltrates skeletal muscle. These lesions are also usually greater than $3 \mathrm{~cm}$ and often shows nuclear beta-catenin reactivity in more than $80 \%$ of cases. Plantar fibromatosis also may raise the differential diagnoses of synovial sarcoma and MPNST. If these are of concern, fluorescence in situ hybridization (SS18) and immunohistochemistry (i.e., S-100, SOX-10, H3K27me3) may be employed to assist in this differential.

\section{TREATMENT AND PROGNOSIS}

Observation is an option for palmar fibromatosis, while excision or incision of the contracture band may be necessary if the contracture results in functional disability and the total flexion deformity is greater than 30 degrees [30] (Figs. 10A, B). Unfortunately, the lesion often recurs. Collagenase (clostridial collagenase histolyticum) has been recently investigated as a treatment option $[8,30,31]$. The disease course seems to cause greater morbidity for white men with a strong family history, those with bilateral involvement, severe disease and ectopic manifestations [32].

As with palmar fibromatosis, conservative measures should be used for plantar fibromatosis prior to recommending surgery. If surgery is performed for symptomatic lesions, complete fasciectomy has fewer recurrences than local and wide excisions
$[8,10,33]$. Along with collagenase, several nonoperative treatments such as steroid injections, verapamil, radiation therapy, extracorporeal shock wave therapy, and tamoxifen have all had variable scientific support [10]. Those with bilateral involvement, multiple nodules, and a positive family history have a worse prognosis [34].

\section{CONCLUSION}

Palmar and plantar fibromatoses are related entities that together form a relatively common diagnostic group encountered in surgical pathology. Further study is needed to ascertain the etiology of these diseases as treatment options currently are limited with a high rate of recurrence. Recognizing the basic clinical, radiographic, and pathologic features is important to arrive at the correct diagnosis.

\section{Ethics Statement}

Not applicable.

\section{Availability of Data and Material}

Data sharing not applicable to this article as no datasets were generated or analyzed during the study.

\section{Code Availability}

Not applicable.

\section{ORCID}

Brian D. Stewart

https://orcid.org/0000-0002-6126-1154

Alessandra F. Nascimento

\section{Author Contributions}

Investigation: BDS. Project administration: BDS. Visualization: BDS, AFN. Writing—original draft: BDS. Writing—review \& editing: BDS, AFN. Approval of final manuscript: all authors. 


\section{Conflicts of Interest}

The authors declare that they have no potential conflicts of interest.

\section{Funding Statement}

No funding to declare.

\section{Acknowledgments}

The authors would like to thank Chung M. Chan, M.D., for the contribution of the clinical photos of palmar fibromatosis.

\section{References}

1. Dibenedetti DB, Nguyen D, Zografos L, Ziemiecki R, Zhou X. Prevalence, incidence, and treatments of Dupuytren's disease in the United States: results from a population-based study. Hand (N Y) 2011; 6: 149-58.

2. Cordova A, Tripoli M, Corradino B, Napoli P, Moschella F. Dupuytren's contracture: an update of biomolecular aspects and therapeutic perspectives. J Hand Surg Br 2005; 30: 557-62.

3. Follonier Castella L, Gabbiani G, McCulloch CA, Hinz B. Regulation of myofibroblast activities: calcium pulls some strings behind the scene. Exp Cell Res 2010; 316: 2390-401.

4. Krause C, Kloen P. Concurrent inhibition of TGF-beta and mitogen driven signaling cascades in Dupuytren's disease: non-surgical treatment strategies from a signaling point of view. Med Hypotheses 2012; 78: 385-8.

5. Ratkaj I, Bujak M, Jurisic D, et al. Microarray analysis of Dupuytren's disease cells: the profibrogenic role of the TGF-beta inducible p38 MAPK pathway. Cell Physiol Biochem 2012; 30: 927-42.

6. Dolmans GH, Werker PM, Hennies HC, et al. Wnt signaling and Dupuytren's disease. N Engl J Med 2011; 365: 307-17.

7. Sayadi LR, Alhunayan D, Sarantopoulos N, et al. The molecular pathogenesis of Dupuytren disease: review of the literature and suggested new approaches to treatment. Ann Plast Surg 2019; 83: 594-600.

8. Carroll P, Henshaw RM, Garwood C, Raspovic K, Kumar D. Plantar fibromatosis: pathophysiology, surgical and nonsurgical therapies: an evidence-based review. Foot Ankle Spec 2018; 11: 168-76.

9. Godette GA, O'Sullivan M, Menelaus MB. Plantar fibromatosis of the heel in children: a report of 14 cases. J Pediatr Orthop 1997; 17: 16-7.

10. Young JR, Sternbach S, Willinger M, Hutchinson ID, Rosenbaum AJ. The etiology, evaluation, and management of plantar fibromatosis. Orthop Res Rev 2019; 11: 1-7.

11. Kim SK, Ioannidis JPA, Ahmed MA, et al. Two genetic variants associated with plantar fascial disorders. Int J Sports Med 2018; 39: 314-21.

12. Strzelczyk A, Vogt H, Hamer HM, Kramer G. Continuous phenobarbital treatment leads to recurrent plantar fibromatosis. Epilepsia 2008; 49: 1965-8.

13. Teh J. Ultrasound of soft tissue masses of the hand. J Ultrason 2012; 12: 381-401.

14. Yacoe ME, Bergman AG, Ladd AL, Hellman BH. Dupuytren's contracture: MR imaging findings and correlation between MR signal intensity and cellularity of lesions. AJR Am J Roentgenol 1993; 160: 813-7.

15. Espert M, Anderson MR, Baumhauer JF. Current concepts review: plantar fibromatosis. Foot Ankle Int 2018; 39: 751-7.
16. McNally EG, Shetty S. Plantar fascia: imaging diagnosis and guided treatment. Semin Musculoskelet Radiol 2010; 14: 334-43.

17. Griffith JF, Wong TY, Wong SM, Wong MW, Metreweli C. Sonography of plantar fibromatosis. AJR Am J Roentgenol 2002; 179: 1167-72.

18. Cohen BE, Murthy NS, McKenzie GA. Ultrasonography of plantar fibromatosis: updated case series, review of the literature, and a novel descriptive appearance termed the "Comb sign". J Ultrasound Med 2018; 37: 2725-31.

19. Narvaez JA, Narvaez J, Ortega R, Aguilera C, Sanchez A, Andia E. Painful heel: MR imaging findings. Radiographics 2000; 20: 333-52.

20. Evans HL. Multinucleated giant cells in plantar fibromatosis. Am J Surg Pathol 2002; 26: 244-8.

21. Carlson JW, Fletcher CD. Immunohistochemistry for beta-catenin in the differential diagnosis of spindle cell lesions: analysis of a series and review of the literature. Histopathology 2007; 51: 509-14.

22. Fausto de Souza D, Micaelo L, Cuzzi T, Ramos ESM. Ledderhose disease: an unusual presentation. J Clin Aesthet Dermatol 2010; 3: 45-7.

23. Wang L, Zhu H. Clonal analysis of palmar fibromatosis: a study whether palmar fibromatosis is a real tumor. J Transl Med 2006; 4: 21.

24. Kaur S, Forsman M, Ryhanen J, Knuutila S, Larramendy ML. No gene copy number changes in Dupuytren's contracture by array comparative genomic hybridization. Cancer Genet Cytogenet 2008; 183: 6-8.

25. De Wever I, Dal Cin P, Fletcher CD, et al. Cytogenetic, clinical, and morphologic correlations in 78 cases of fibromatosis: a report from the CHAMP Study Group. CHromosomes And Morphology. Mod Pathol 2000; 13: 1080-5.

26. Shih B, Tassabehji M, Watson JS, Bayat A. DNA copy number variations at chromosome 7p14.1 and chromosome 14q11.2 are associated with Dupuytren's disease: potential role for MMP and Wnt signaling pathway. Plast Reconstr Surg 2012; 129: 921-32.

27. Michou L, Lermusiaux JL, Teyssedou JP, Bardin T, Beaudreuil J, PetitTeixeira E. Genetics of Dupuytren's disease. Joint Bone Spine 2012; 79: 7-12.

28. Breiner JA, Nelson M, Bredthauer BD, Neff JR, Bridge JA. Trisomy 8 and trisomy 14 in plantar fibromatosis. Cancer Genet Cytogenet 1999; 108: 176-7.

29. Sawyer JR, Sammartino G, Gokden N, Nicholas RW. A clonal reciprocal $\mathrm{t}(2 ; 7)(\mathrm{p} 13 ; \mathrm{p} 13)$ in plantar fibromatosis. Cancer Genet Cytogenet 2005; 158: 67-9.

30. Denkler KA, Vaughn CJ, Dolan EL, Hansen SL. Evidence-based medicine: options for Dupuytren's contracture: incise, excise, and dissolve. Plast Reconstr Surg 2017; 139: 240e-55e.

31. Sanjuan-Cervero R. Current role of the collagenase Clostridium histolyticum in Dupuytren's disease treatment. Ir J Med Sci 2020; 189: 529-34.

32. Townley WA, Baker R, Sheppard N, Grobbelaar AO. Dupuytren's contracture unfolded. BMJ 2006; 332: 397-400.

33. van der Veer WM, Hamburg SM, de Gast A, Niessen FB. Recurrence of plantar fibromatosis after plantar fasciectomy: single-center long-term results. Plast Reconstr Surg 2008; 122: 486-91.

34. Kadir HK, Chandrasekar CR. Partial fasciectomy is a useful treatment option for symptomatic plantar fibromatosis. Foot (Edinb) 2017; 31: 31-4. 